\title{
ASSOCIATED CURVES OF FRENET CURVES IN THREE DIMENSIONAL COMPACT LIE GROUP
}

\author{
S. KIZILTUǦ AND M. ÖNDER \\ Received 09 September, 2014
}

\begin{abstract}
General definition of associated curves of a Frenet curve is given in a three dimensional compact Lie group $G$. The principal normal direction curve and principal normal donor curve are introduced and some characterizations for these curves are obtained in $G$. Later, the relationships between a principal normal direction curve and some special curves such as helix, slant helix or curve with a special torsion are obtained.
\end{abstract}

2010 Mathematics Subject Classification: 53A04; 22E15

Keywords: Lie group, associated curve, donor curve

\section{INTRODUCTION}

The curve theory in the Euclidean space or in a Riemannian manifold has been developed by many mathematicians so far by the view of local or global differential geometry. The most fascinating subject of curve theory is to obtain some characterizations for a regular curve or curve pairs. The well-known characterizations obtained for a single curve have allowed to define some special curves such as helix, slant helix, plane curve, spherical curve, etc. [1,11,13,20,23] and these special curves, especially helices, are used in many applications [2, 9, 10, 19]. Similarly, by considering two curves, some special curve pairs such as involute-evolute curves, Bertrand curves, Mannheim curves have been defined and studied so far $[4,12,14,15,18,21,22]$.

Furthermore, Çöken and Çiftçi have studied the degenerate semi-Riemannian geometry of a Lie group [6]. They have obtained a naturally reductive homogeneous semi-Riemannian space using the Lie group. Later, some of subjects given above have been considered in three dimensional Lie groups and some characterizations for these curves have been obtained in a three dimensional Lie group [3,5, 16-18].

Recently, Choi and Kim have defined a new curve pair in the Euclidean 3-space $E^{3}$ [7]. They have considered a unit vector field $X$ defined in the Frenet basis of a curve $\alpha$ and an integral curve $\gamma$ of $X$ and given the definition and characterizations 
of principal-direction curve and principal-donor curve in $E^{3}$. They have also given some applications of these curves related to a helix, a slant helix or a plane curve.

In the present paper, we give the general definition of associated curves of a Frenet curve in a three dimensional compact Lie group $G$. Then, we define principaldirection curve and principal-donor curve in $G$ and obtain some characterizations for these curves. In the last section of the paper, we give the relationships between principal direction curves and some special curves such as helix, slant helix or rectifying curve in $G$.

\section{Preliminaries}

Let $G$ be a Lie group with a bi-invariant metric $\langle$,$\rangle and D$ be the Levi-Civita connection of $G$. It is well-known that the Lie algebra $g$ of $G$ is isomorphic to $T_{e} G$ where $e$ is neutral element of $G$. Let $\langle$,$\rangle be a bi-invariant metric on G$. Then we have

and

$$
\langle X,[Y, Z]\rangle=\langle[X, Y], Z\rangle
$$

$$
D_{X} Y=\frac{1}{2}[X, Y]
$$

for all $X, Y, Z \in g[5]$.

Let $\alpha: I \subset \mathrm{R} \rightarrow G$ be an arc-lenghted curve and $\left\{X_{1}, X_{2}, \ldots, X_{n}\right\}$ be an orthonormal basis of $g$. In this case, we can write any two vector fields $W$ and $Z$ along the curve $\alpha$ as $W=\sum_{i=1}^{n} w_{i} X_{i}$ and $Z=\sum_{i=1}^{n} z_{i} X_{i}$ where $w_{i}: I \rightarrow \mathrm{R}$ and $z_{i}: I \rightarrow \mathrm{R}$ are smooth functions. Also the Lie bracket of two vector fields $W$ and $Z$ is given by

$$
[W, Z]=\sum_{i=1}^{n} w_{i} z_{i}\left[X_{i}, X_{j}\right],
$$

and the covariant derivative of $W$ along the curve $\alpha$ with the notation $D_{\alpha^{\prime}} W$ is given as follows

$$
D_{\alpha^{\prime}} W=\dot{W}+\frac{1}{2}[T, W],
$$

where $T=\alpha^{\prime}$ and $\dot{W}=\sum_{i=1}^{n} \dot{w}_{i} X_{i}$ [8]. If $W$ is the left-invariant vector field to the curve $\alpha$ then it is important to point out that $\dot{W}=0$. Let $G$ be a three dimensional Lie group and $(T, N, B, \kappa, \tau)$ denote the Frenet apparatus of the curve $\alpha$. Then the Frenet formulae of $\alpha$ are given as follows

$$
D_{T} T=\kappa N, D_{T} N=-\kappa T+\tau B, D_{T} B=-\tau N
$$

where $\kappa=\|\dot{T}\|$. If $\kappa=\|\dot{T}\| \neq 0$, then $\alpha$ is called a Frenet curve in $G$ [16]. 
Proposition 1 ([17]). Let $\alpha: I \subset \mathrm{R} \rightarrow G$ be an arc length parametrized curve with the Frenet apparatus $(T, N, B)$. Then the following equalities hold

$$
\left\{\begin{array}{l}
{[T, N]=\langle[T, N], B\rangle B=2 \tau_{G} B} \\
{[T, B]=\langle[T, B], N\rangle N=-2 \tau_{G} N .}
\end{array}\right.
$$

Let $\alpha: I \subset \mathrm{R} \rightarrow G$ be an arc length parametrized curve with arc length parameter $s$. Then from equation (2.3) and Proposition 2.1, the following Frenet formulae are obtained

$$
\left(\begin{array}{l}
\frac{d T}{d s} \\
\frac{d N}{d s} \\
\frac{d B}{d s}
\end{array}\right)=\left(\begin{array}{lll}
0 & \kappa & 0 \\
-\kappa & 0 & \tau-\tau_{G} \\
0 & -\left(\tau-\tau_{G}\right) & 0
\end{array}\right)\left(\begin{array}{l}
T \\
N \\
B
\end{array}\right),
$$

where $\{T, N, B\}$ is the Frenet frame $\tau_{G}=\frac{1}{2}\langle[T, N], B\rangle$ and $\kappa, \tau$ are curvature and torsion of $\alpha$ in three dimensional Lie group $G$, respectively [17].

Definition 1 ([17]). Let $\alpha: I \subset \mathrm{R} \rightarrow G$ be an arc length parametrized curve in three dimensional compact Lie group with the Frenet apparatus $(T, N, B, \kappa, \tau)$. Then the harmonic curvature function of the curve $\alpha$ is defined by

$$
H=\frac{\tau-\tau_{G}}{\kappa} \text {. }
$$

Theorem 1 ([5]). Let $\alpha: I \subset \mathrm{R} \rightarrow G$ be an arc length parametrized curve in three dimensional compact Lie group with the Frenet apparatus $(T, N, B, \kappa, \tau)$. The curve $\alpha$ is a general helix if and only if $\tau=c \kappa+\tau_{G}$, where $c$ is a constant.

From Definition 1 and Theorem 1, we have the following corollary:

Corollary 1. The curve $\alpha: I \subset \mathrm{R} \rightarrow G$ is a general helix in $G$ if and only if $H=$ constant.

Theorem 2 ([17]). Let $\alpha: I \subset \mathrm{R} \rightarrow G$ be an arc length parametrized curve with the Frenet apparatus $(T, N, B, \kappa, \tau)$. Then $\alpha$ is a slant helix if and only if the function

$$
\sigma=\frac{\kappa\left(1+H^{2}\right)^{3 / 2}}{H^{\prime}}
$$

is a constant where $H$ is the harmonic curvature function of the curve $\alpha$.

Theorem 3 ([16]). Let $\alpha: I \subset \mathrm{R} \rightarrow G$ be an arc length parametrized Bertrand curve whose curvature function $\kappa$ and harmonic curvature function $H$ satisfy $\lambda \kappa(s)+$ $\mu \kappa(s) H(s)=1$, for all $s \in I$, where $\lambda, \mu$ are constants. If the curve $\beta(s)$ given by $\beta(s)=\alpha(s)+\lambda N(s)$ for all $s \in I$ is a special Frenet curve, then $(\alpha, \beta)$ is the Bertrand curve couple. 


\section{THE PRINCIPAL-DIRECTION CURVES AND THE PRINCIPAL-DONOR CURVES IN A COMPACT LIE GROUP}

In this section, we study the principal-direction curves and the principal-donor curves in three dimensional compact Lie group $G$ with a bi-invariant metric. First, we give the existence and definition of an $W$-direction curve of a curve in a Riemannian manifold.

Theorem 4. Let $M$ be an n-dimensional Riemannian manifold and $\alpha(t): I \rightarrow$ $U \subset M$ a curve on a local chart $U$ of $M$. Then, for a continuous vector field $W=$ $W(t)$ on $U \subset M$ along the curve $\alpha$, there exists a curve $\gamma(t): I \subset \mathrm{R} \rightarrow M$ on the neighborhood $U$ such that $\gamma^{\prime}(t)=W(t)$.

Proof. Let $x=\left(x_{1}, x_{2}, \ldots x_{n}\right)$ be a local coordinate on $U$. Then the vector field $W=W(t)$ on $U$ along $\gamma(t)$ is expressed by $\left.\sum_{i=1}^{n} W_{i}(t) \frac{\partial}{\partial x_{i}}\right|_{\gamma(t)}$ where $W_{i}(t), \quad(1 \leq$ $i \leq n)$ are continuous real valued functions on the interval $I$. Let $x_{i}: I \rightarrow \mathrm{R}$ be a solution of the linear ordinary differential equation $\frac{\partial}{\partial t} x_{i}(t)=W_{i}(t)$, for each $i$. Then, the curve $\gamma$ is clearly $x^{-1} \circ\left(x_{1}(t), x_{2}(t), \ldots, x_{n}(t)\right)$.

Remark 1. Let $\alpha(t): I \rightarrow U \subset M$ be a curve in an $n$-dimensional Riemannian manifold $M$ and $W=W(t)$ a vector field on $U \subset M$ along the curve $\alpha$. Then, for a point $p=\alpha\left(t_{0}\right)$, there are a sub-interval $J \subset I$ containing $t_{0}$ such that the unique curve $\gamma(t)$ on a local neighborhood $U$ satisfying $\gamma\left(t_{0}\right)=p$ and $\frac{d}{d t} \gamma(t)=\left.W(t)\right|_{U}$ for all $t \in J$.

Definition 2. For a given curve in an $n$-dimensional Riemannian manifold $M$ and a point $p$ on $\alpha$, the curve $\gamma$ of Remark 3.2 is called the $W$-direction curve passing the point $p$ of $\alpha$. In this case, $\alpha$ is called the $W$-donor curve passing $p$ of $\gamma$.

Moreover, it is well-known that a Lie group is also a differentiable manifold, with the property that the group operations are compatible with the smooth structure. Then, Definition 2 can be considered in a compact Lie group $G$. For this purpose, let consider a Frenet curve $\alpha$ in three dimensional compact Lie group $G$ with the Frenet frame $\{T, N, B\}$ and a unit vector field $W$ given by

$$
W(s)=\eta(s) T(s)+\vartheta(s) N(s)+\xi(s) B(s),
$$

where $\eta, \vartheta$ and $\xi$ are arbitrary differentiable functions of $s$ which is the arc length parameter of $\alpha$. Let

$$
\eta^{2}(s)+\vartheta^{2}(s)+\xi^{2}(s)=1,
$$

holds. Then, we define a $W$-direction curve and a $W$-donor curve in three dimensional compact Lie group as follows. 
Definition 3. Let $\alpha$ be a Frenet curve in a three dimensional compact Lie group $G$ and $W$ be a unit vector field satisfying the equations (3.1) and (3.2). The integral curve $\gamma: I \subset \mathrm{R} \rightarrow G$ of $W$ is called a $W$-direction curve of $\alpha$. The curve $\alpha$ whose $W$-direction curve is $\gamma$ is called the $W$-donor curve in three dimensional compact Lie group $G$.

Definition 4. An integral curve of $N(s)$ (resp. $B(s)$ ) in (3.1) is called the principaldirection curve (resp. binormal-direction curve) of $\alpha$ in three dimensional compact Lie group $G$.

Remark 2. A principal-direction (resp. the binormal-direction) curve is an integral curve of $W(s)$ with $\eta(s)=\xi(s)=0, \vartheta(s)=1(\operatorname{resp} . \eta(s)=\vartheta(s)=0, \xi(s)=1)$ for all $s$ in (3.1).

Proposition 2. Let $\alpha: I \subset \mathrm{R} \rightarrow G$ be a Frenet curve and an integral curve of $W(s)$ be the curve $\gamma: I \subset \mathrm{R} \rightarrow G$. Then, the principal-direction curve of $\gamma$ equals to $\alpha$ if and only if

$$
\eta(s)=0, \vartheta(s)=\sin \left(\int H \kappa d s\right) \neq 0, \quad \xi(s)=\cos \left(\int H \kappa d s\right) \neq 0,
$$

hold, where $H$ is harmonic curvature function of the curve $\alpha$.

Proof. Let $\alpha: I \subset \mathrm{R} \rightarrow G$ be an arc length parametrized curve with the Frenet apparatus $\{T, N, B\}$. From (3.2) we have

$$
\eta^{2}(s)+\vartheta^{2}(s)+\xi^{2}(s)=1 .
$$

Differentiating (3.4) with respect to $s$ gives us

$$
\eta \eta^{\prime}+\vartheta \vartheta^{\prime}+\xi \xi^{\prime}=0
$$

Also, differentiating (3.1) with respect to $s$ and using (2.3) we get

$$
\begin{aligned}
& \frac{d}{d s} W(s)=\eta^{\prime}(s) T(s)+\vartheta^{\prime}(s) N(s)+\xi^{\prime}(s) B(s) \\
& \quad+\eta(s)\left(D_{T} T-\frac{1}{2}[T, T]\right)+\vartheta(s)\left(D_{T} N-\frac{1}{2}[T, N]\right) \\
& \quad+\xi(s)\left(D_{T} B-\frac{1}{2}[T, B]\right)
\end{aligned}
$$

By using the Frenet formulas and with the aid of equation (2.5), we obtain

$$
\frac{d}{d s} W=\left(\eta^{\prime}-\vartheta \kappa\right) T+\left(\vartheta^{\prime}+\eta \kappa-\xi H \kappa\right) N+\left(\xi^{\prime}+\vartheta H \kappa\right) B,
$$

where $H$ is the harmonic curvature function and $\kappa$ is the curvature of $\alpha$. Since

$$
\frac{d}{d s} W(s)=\frac{d^{2}}{d s^{2}} \gamma(s)=\dot{\bar{T}}(s)=D_{\bar{T}} \bar{T}-\frac{1}{2}[\bar{T}, \bar{T}]=\bar{\kappa} \bar{N},
$$


$\alpha$ is a principal-direction curve of $\gamma$ if and only if the following system hold

$$
\left\{\begin{array}{l}
\eta^{\prime}-\vartheta \kappa \neq 0, \\
\vartheta^{\prime}+\eta \kappa-\xi H \kappa=0, \\
\xi^{\prime}+\vartheta H \kappa=0 .
\end{array}\right.
$$

Multiplying the last equation of this system by $\xi$ and substituting the obtained result in (3.5) gives us

$$
\eta \eta^{\prime}+\vartheta \vartheta^{\prime}=\xi \vartheta H \kappa .
$$

Similarly, multiplying the second equation of the system by $\vartheta$ and using the last equality we have $\eta\left(\eta^{\prime}-\vartheta \kappa\right)=0$. Since $\eta^{\prime}-\vartheta \kappa \neq 0$, it follows that $\eta=0$. Then, from the system we have

and it has the following solution

$$
\left\{\begin{array}{l}
\xi^{\prime}+\vartheta H \kappa=0 \\
\vartheta^{\prime}-\xi H \kappa=0
\end{array}\right.
$$

$$
\vartheta(s)=\sin \left(\int H \kappa d s\right) \neq 0, \quad \xi(s)=\cos \left(\int H \kappa d s\right) \neq 0 .
$$

Definition 5. An integral curve $\gamma$ of

$$
W(s)=\sin \left(\int H \kappa d s\right) N(s)+\cos \left(\int H \kappa d s\right) B(s)
$$

is called a principal-donor curve of $\alpha$.

Theorem 5. Let $\alpha: I \subset \mathrm{R} \rightarrow G$ be an arc length parametrized curve with the Frenet apparatus $(T, N, B, \kappa, \tau)$ and $\gamma$ the principal-direction curve of $\alpha$. Then the curvature $\bar{\kappa}$ and torsion $\bar{\tau}$ of $\gamma$ are given by

$$
\bar{\kappa}=\sqrt{\kappa^{2}\left(1+H^{2}\right)}, \quad \bar{\tau}-\bar{\tau}_{g}=-\frac{H^{\prime}}{1+H^{2}},
$$

where $H$ is harmonic curvature function of the curve $\alpha$.

Proof. By the definition of principal direction curve of $\alpha$,

$$
\frac{d \gamma}{d s}=\bar{T}=N
$$

Differentiating (3.9) with respect to $s$ and considering Frenet formulas, we get

$$
\bar{\kappa} \bar{N}=-\kappa T+\kappa H B .
$$


From the norms of both sides in (3.10) we have

$$
\bar{\kappa}=\sqrt{\kappa^{2}\left(1+H^{2}\right)} .
$$

Substituting (3.11) into (3.10), we obtain

and then

$$
\bar{N}=-\frac{1}{\sqrt{1+H^{2}}} T+\frac{H}{\sqrt{1+H^{2}}} B,
$$

$$
\bar{B}=\bar{T} \times \bar{N}=N \times \bar{N}=\frac{H}{\sqrt{1+H^{2}}} T-\frac{1}{\sqrt{1+H^{2}}} B .
$$

Differentiating (3.12) with respect to $s$ we have

$$
\dot{\bar{B}}=\frac{H^{\prime}}{\left(1+H^{2}\right)^{\frac{3}{2}}}(T-H B) .
$$

Since $\dot{\bar{B}}=D_{\bar{T}} \bar{B}-\frac{1}{2}[\bar{T}, \bar{B}]=\left(\bar{\tau}_{g}-\bar{\tau}\right) \bar{N}$, from (3.13) it follows

$$
\left(\bar{\tau}_{g}-\bar{\tau}\right) \bar{N}=\frac{H^{\prime}}{\left(1+H^{2}\right)^{\frac{3}{2}}}(T-H B),
$$

which gives us

$$
\bar{\tau}-\bar{\tau}_{g}=-\frac{H^{\prime}}{1+H^{2}} .
$$

From Theorem 5, we have the following corollaries:

Corollary 2. Let $\alpha: I \subset \mathrm{R} \rightarrow G$ be a Frenet curve in three dimensional compact Lie Group $G$. If $\gamma$ is the principal-direction curve of $\alpha$, then the relationships between the Frenet frames of the curves are given as follows

$$
\bar{T}=N, \quad \bar{N}=-\frac{1}{\sqrt{1+H^{2}}} T+\frac{H}{\sqrt{1+H^{2}}} B, \quad \bar{B}=\frac{H}{\sqrt{1+H^{2}}} T-\frac{1}{\sqrt{1+H^{2}}} B .
$$

Corollary 3. Let $\alpha: I \subset \mathrm{R} \rightarrow G$ be a Frenet curve in three dimensional compact Lie Group G. If $\gamma$ is the principal-direction curve of $\alpha$, then

$$
\bar{H}=-\frac{H^{\prime}}{\kappa\left(1+H^{2}\right)^{3 / 2}} .
$$


Theorem 6. Let $\alpha: I \subset \mathrm{R} \rightarrow G$ be a Frenet curve. If $\alpha$ is principal donor curve of the curve $\gamma$, then the curvature $\kappa$ and the torsion $\tau-\tau_{G}$ of the curve $\alpha$ are given by

$$
\kappa=\bar{\kappa}\left|\sin \left(\int \bar{H} \bar{\kappa} d s\right)\right|, \quad \tau-\tau_{G}=\bar{\kappa}\left|\cos \left(\int \bar{H} \bar{\kappa} d s\right)\right|,
$$

recpevtively.

Proof. Since $\alpha$ is a principal-donor curve of $\gamma$, from Definition 3, we have

$$
\frac{d \alpha(s)}{d s}=\sin \left(\int \bar{H} \bar{\kappa} d s\right) \bar{N}(s)+\cos \left(\int \bar{H} \bar{\kappa} d s\right) \bar{B}(s) .
$$

Differentiating (3.16) with respect to $s$ we have

$$
\frac{d^{2} \alpha(s)}{d s^{2}}=-\bar{\kappa} \sin \left(\int \bar{H} \bar{\kappa} d s\right) \bar{T}(s) .
$$

Since $\left\|\frac{d^{2} \alpha(s)}{d s^{2}}\right\|=\kappa$, we get

$$
\kappa=\bar{\kappa}\left|\sin \left(\int \bar{H} \bar{\kappa} d s\right)\right| .
$$

Then substituting (3.18) in (3.10), it follows that

$$
\tau-\tau_{G}=\bar{\kappa}\left|\cos \left(\int \bar{H} \bar{\kappa} d s\right)\right| .
$$

Hence our theorem is proved.

\section{APPLICATIONS OF PRINCIPAL-DIRECTION CURVES AND PRINCIPAL DONOR CURVES TO SOME SPECIAL CURVES IN A COMPACT LIE GROUP}

In this section, we give relationships between the principal-direction curve, the principal donor curve and some special curves such as helix or slant helix in a three dimensional compact Lie group $G$.

Let $\gamma$ be a principal-direction curve of $\alpha$ and $\bar{\gamma}$ principal-direction curve of $\gamma$ in $G$. Then, $\bar{\gamma}$ is called a second principal-direction curve of $\alpha$ and $\alpha$ is called a second principal-donor curve of $\bar{\gamma}$.

Theorem 7. Let $\alpha: I \subset \mathrm{R} \rightarrow G$ be a Frenet curve in $G$. Then the followings are equivalent:

i) A Frenet curve $\alpha$ is a general helix in $G$.

ii) $\alpha$ is a principal-donor curve of a curve $\gamma$ with curvature $\bar{\tau}=\bar{\tau}_{g}$ and torsion $\bar{\kappa}=\frac{\sqrt{1+c^{2}}}{c}\left|\tau-\tau_{G}\right|$ in $G$ where $c=H$ is a constant

iii) A principal-direction curve of $\alpha$ is a curve $\gamma$ with curvature $\bar{\tau}=\bar{\tau}_{g}$ in $G$. 
Let $\bar{\gamma}=(x(s), y(s), 0)$ be a unit speed curve with curvature $\bar{\tau}=\bar{\tau}_{g}$ in $G$. Then $\bar{\gamma}$ can be expressed by

$$
x(s)=\int_{0}^{s} \cos \left(\int_{0}^{\sigma} \overline{\bar{\kappa}}(t) d t\right) d \sigma, y(s)=\int_{0}^{s} \sin \left(\int_{0}^{\sigma} \overline{\bar{\kappa}}(t) d t\right) d \sigma,
$$

where $\overline{\bar{\kappa}}=\sqrt{\left[x^{\prime \prime}(s)\right]^{2}+\left[y^{\prime \prime}(s)\right]^{2}}$ is the curvature of $\bar{\gamma}$. Denote the Frenet frame of the curve $\bar{\gamma}$ by $\{\overline{\bar{T}}, \overline{\bar{N}}, \overline{\bar{B}}\}$. Then we have

$$
\begin{aligned}
& \overline{\bar{T}}(s)=\left(\cos \left(\int_{0}^{s} \overline{\bar{\kappa}}(t) d t\right), \sin \left(\int_{0}^{s} \overline{\bar{\kappa}}(t) d t\right), 0\right), \\
& \overline{\bar{N}}(s)=\left(-\sin \left(\int_{0}^{s} \overline{\bar{\kappa}}(t) d t\right), \cos \left(\int_{0}^{s} \overline{\bar{\kappa}}(t) d t\right), 0\right), \\
& \overline{\bar{B}}(s)=(0,0,1) .
\end{aligned}
$$

Thus, we construct a principal-donor curve of $\bar{\gamma}$ as:

$$
=\int_{0}^{s} \gamma^{\prime}(s) d s=\left(a \int_{0}^{s} \sin \left(\int_{0}^{\sigma} \overline{\bar{\kappa}}(t) d t\right) d \sigma,-a \int_{0}^{s} \cos \left(\int_{0}^{\sigma} \overline{\bar{\kappa}}(t) d t\right) d \sigma, b s\right)
$$

where $a \neq 0$ and $b$ are constant with $a^{2}+b^{2}=1$. Consequently, we have the following corollary:

Corollary 4. Let $\bar{\gamma}=(x(s), y(s), 0)$ be a unit speed curve with curvature $\bar{\tau}=\bar{\tau}_{g}$ in $G$. Then, the principal-donor curve $\gamma$ of $\bar{\gamma}$ is given by

where $a \neq 0$ and $b$ are constant with $a^{2}+b^{2}=1$.

$$
\gamma=(a y(s),-a x(s), b s),
$$

From Theorem 2 and Corollary 2 we have the following theorem

Theorem 8. Let $\alpha: I \subset \mathrm{R} \rightarrow G$ be a Frenet curve in $G$. Then the followings are equivalent:

i) A frenet curve $\alpha$ is a slant helix in $G$.

ii) $\alpha$ is a principal-donor curve of a general helix in $G$.

iii) $\alpha$ is a second principal-donor curve of a curve $\gamma$ with curvature $\bar{\tau}=\bar{\tau}_{g}$ in $G$.

iv) A principal-direction curve of $\alpha$ is a general helix in $G$.

v) A second principal-direction curve of $\alpha$ is a curve $\gamma$ with curvature $\bar{\tau}=\bar{\tau}_{g}$ in $G$.

Definition 6 ([3]). Let $\alpha: I \subset \mathrm{R} \rightarrow G$ be an arc length parametrized curve in three dimensional compact Lie group $G$. If the position vector of $\alpha$ always lies in its rectifying plane, then it is called a rectifying curve in $G$.

Definition 7. Let $\alpha: I \subset \mathrm{R} \rightarrow G$ be an arc length parametrized curve in three dimensional compact Lie group $G$ and the curve $\gamma$ the principal-direction curve of $\alpha$. If the position vector of $\gamma$ always lies in rectifying plane of $\alpha$, then $\gamma$ is called principal direction rectifying (or $P D$-rectifying) curve in $G$. 
By this definition, the position vector of $P D$-rectifying curve $\gamma$ in $G$ satisfies the following equation

$$
\gamma=\omega(s) T(s)+\rho(s) B(s),
$$

where $\omega(s), \rho(s)$ are non-zero smooth functions and $T(s), B(s)$ are Frenet vectors of $\alpha$. Since $\alpha$ is a principal-donor curve of $\gamma$ in $G$, from Definition (3.8), we have

$$
\left\{\begin{array}{l}
\alpha^{\prime}(s)=T(s)=\sin \left(\int \bar{H} \bar{\kappa} d s\right) \bar{N}(s)+\cos \left(\int \bar{H} \bar{\kappa} d s\right) \bar{B}(s), \\
B(s)=-\cos \left(\int \bar{H} \bar{\kappa} d s\right) \bar{N}(s)+\sin \left(\int \bar{H} \bar{\kappa} d s\right) \bar{B}(s) .
\end{array}\right.
$$

Then substituting (4.2) in (4.1) it follows

$$
\begin{aligned}
& \gamma=\left[\omega(s) \sin \left(\int \bar{H} \bar{\kappa} d s\right)-\rho(s) \cos \left(\int \bar{H} \bar{\kappa} d s\right)\right] \bar{N}(s) \\
& \quad+\left[\omega(s) \cos \left(\int \bar{H} \bar{\kappa} d s\right)+\rho(s) \sin \left(\int \bar{H} \bar{\kappa} d s\right)\right] \bar{B}(s) .
\end{aligned}
$$

Differentiating (4.3) with respect to $s$ gives us

From the last equality we have

$$
\begin{aligned}
\bar{T}= & \bar{\kappa}\left[-\omega \sin \left(\int \bar{H} \bar{\kappa} d s\right)-\rho \cos \left(\int \bar{H} \bar{\kappa} d s\right)\right] \bar{T}(s) \\
& +\left[\omega^{\prime} \sin \left(\int \bar{H} \bar{\kappa} d s\right)-\rho^{\prime} \cos \left(\int \bar{H} \bar{\kappa} d s\right)\right] \bar{N}(s) \\
& +\left[\omega^{\prime} \cos \left(\int \bar{H} \bar{\kappa} d s\right)+\rho^{\prime} \sin \left(\int \bar{H} \bar{\kappa} d s\right)\right] \bar{B}(s) .
\end{aligned}
$$

$$
\omega^{\prime}(s)=\rho^{\prime}(s)=0, \omega \bar{\kappa} \sin \left(\int \bar{H} \bar{\kappa} d s\right)+\rho \bar{\kappa} \cos \left(\int \bar{H} \bar{\kappa} d s\right)=-1 .
$$

Since $\omega$ and $\rho$ are non-zero constants, the last equation implies that

$$
\bar{\tau}-\bar{\tau}_{G}=\frac{\bar{\kappa}^{\prime}}{\bar{\kappa} \sqrt{\left(\omega^{2}+\rho^{2}\right) \bar{\kappa}^{2}-1}} .
$$

Moreover, by writing (3.15) in (28) we have

$$
\lambda \kappa+\mu \kappa H=1 .
$$

where $\lambda=-\omega, \mu=-\rho$ are constants. Then we can give the following theorem characterizing $P D$-rectifying curves in $G$.

Theorem 9. Let $\gamma$ be a Frenet curve in three dimensional compact Lie group $G$ and $\alpha$ a principal-donor curve of $\gamma$. Then, the followings are equivalent,

i) gamma is $P D$-rectifying curve in $G$.

ii) $\gamma$ is a Frenet curve with non-zero curvature and non-zero torsion satisfying equation (4.4).

iii) The position vector of $\gamma$ lies in its normal plane in $G$.

iv) There exists a Bertrand couple $(\alpha, \beta)$ such that $\beta(s)=\alpha(s)+\lambda N(s)$ for all $s \in I$ is a special Frenet curve in $G$. 


\section{REFERENCES}

[1] M. Barros, "General helices and a theorem of lancret," Proc. Amer. Math. Soc., vol. 125, no. 5, pp. 1503-1509, 1997, doi: 10.1090/S0002-9939-97-03692-7.

[2] J. Beltran and J. Monterde, "A characterization of quintic helices," J. Comput. Appl. Math., vol. 206, no. 1, pp. 116-121, 2007, doi: 10.1016/j.cam.2006.06.001.

[3] Z. Bozkurt, I. Gök, O. Okuyucu, and F. Ekmekçi, "Characterizations of rectifying, normal and osculating curves in three dimensional compact lie groups," Life Science Journal, vol. 10, no. 3 , pp. 819-823, 2013.

[4] J. Burke, "Bertrand curves associated with a pair of curves," Mathematics Magazine, vol. 34, no. 1, pp. 60-62, 1960, doi: 10.2307/2687860.

[5] U. Çiftçi, "A generalization of lancret's theorem," Journal of Geometry and Physics, vol. 59, no. 12 , pp. 1597-1603, 2009.

[6] A. Çöken and U. Çiftçi, "A note on the geometry of lie groups," Nonlinear Analysis TMA, vol. 68, no. 7, pp. 2013-2016, 2008.

[7] J. Choi and Y. Kim, "Associated curves of a frenet curve and their applications," Applied Mathematics and Computation, vol. 218, no. 18, pp. 9116-9124, 2012, doi: 10.1016/j.amc.2012.02.064.

[8] P. Crouch and F. Silva Leite, "The dynamic interpolation problem: on riemannian manifolds, lie groups, and symmetric spaces," J. Dynam. Control Systems, vol. 1, no. 2, pp. 177-202, 1995, doi: 10.1007/BF02254638.

[9] A. Da Fonseca and C. Malta, "Lancret helices," arXiv:physics/0507105v1 [physics.bio-ph], 2005.

[10] R. Farouki, C. Han, C. Manni, and A. Sestini, "Characterization and construction of helical polynomial space curves," J. Comput. Appl. Math., vol. 162, no. 2, pp. 365-392, 2004, doi: 10.1016/j.cam.2003.08.030.

[11] H. Hayden, "On a generalized helix in a riemannian n-space," Proc. Lond. Math. Soc., vol. 32, pp. 337-345, 1931, doi: 10.1112/plms/s2-32.1.337.

[12] S. Izumiya and N. Takeuchi, "Generic properties of helices and bertrand curves," Journal of Geometry, vol. 74, no. 1-2, pp. 97-109, 2002, doi: 10.1007/PL00012543.

[13] S. Izumiya and N. Takeuchi, "New special curves and developable surfaces," Turk. J. Math., vol. 28, pp. 153-163, 2004.

[14] T. Kahraman, M. Önder, M. Kazaz, and H. Uğurlu, "Some characterizations of mannheim partner curves in minkowski 3-space," Proceedings of the Estonian Academy of Sciences, vol. 60, no. 4, pp. 210-220, 2011, doi: 10.3176/proc.2011.4.02.

[15] H. Liu and F. Wang, "Mannheim partner curves in 3-space," Journal of Geometry, vol. 88, no. 1-2, pp. 120-126, 2008, doi: 10.1007/s00022-007-1949-0.

[16] O. Okuyucu, İ. Gök, Y. Yaylı, and F. Ekmekçi, "Bertrand curves in three dimensional lie groups," arxiv: 1211.6424v1 [Math.DG], 2012.

[17] O. Okuyucu, İ. Gök, Y. Yaylı, and F. Ekmekçi, "Slant helices in three dimensional lie groups," Applied Mathematics and Computation, vol. 221, no. 15, pp. 672-683, 2013, doi: 10.1016/j.amc.2013.07.008.

[18] O. Okuyucu, O. Yıldız, and M. Tosun, "Spinor frenet equations in three dimensional lie groups," arxiv: 1212.6560v1 [Math.DG], 2012.

[19] J. Puig-Pey, A. Gálvez, and A. Iglesias, Helical curves on surfaces for computer-aided geometric design and manufacturing, ser. Lecture Notes in Comput. Sci.,. Berlin: Springer, 2004, vol. 3044, doi: 10.1007/978-3-540-24709-8'81.

[20] D. Struik, Lectures on Classical Differential Geometry, 2nd ed. Dover: Addison Wesley, 1950.

[21] F. Wang and H. Liu, "Mannheim partner curves in 3-euclidean space," Mathematics in Practice and Theory, vol. 37, no. 1, pp. 141-143, 2007. 
[22] J. Whittemore, "Bertrand curves and helices," Duke Math. J., vol. 6, no. 1, pp. 235-245, 1940, doi: 10.1215/S0012-7094-40-00618-4.

[23] Y. Wong, "On the generalized helices of hayden and syptak in an n-space," Proc. Cambridge Philos. Soc., vol. 37, pp. 229-243, 1941, doi: 10.1017/S0305004100021721.

Authors' addresses

\section{S. Kızıltů̆}

Erzincan University, Faculty of Arts and Sciences, Department of Mathematics, Erzincan, Turkey E-mail address: skiziltug@erzincan.edu.tr

\section{M. Önder}

Celal Bayar University, Faculty of Arts and Sciences, Department of Mathematics, Muradiye Campus, 45140 Manisa, Turkey

E-mail address: mehmet.onder@cbu.edu.tr 